\title{
Direction Dependent Diffusion of Aligned Magnetic Rods by Means of X-Ray Photon Correlation Spectroscopy
}

\author{
Joachim Wagner* \\ Institut für Chemie, Universität Rostock, Albert-Einstein-Straße 3a, 18059 Rostock, Germany \\ Christian Märkert \\ Physikalische Chemie, Universität des Saarlandes, Campus B22, 66123 Saarbrücken, Germany \\ Birgit Fischer and Leonard Müller \\ Deutsches Elektronensynchrotron, Notkestraße 85, 22607 Hamburg, Germany
}

(Received 17 September 2012; published 23 January 2013)

\begin{abstract}
Rodlike hematite particles in suspension align perpendicular to an external magnetic field due to a negative anisotropy of their magnetic susceptibility $\Delta \chi$. The diffusion tensor consists of two principal constants $D_{\|}$and $D_{\perp}$ for the diffusion parallel and perpendicular to the long particle axis. X-ray photon correlation spectroscopy is capable of probing the diffusive motion in optically opaque suspensions of rodlike hematite particles parallel to the direction of the scattering vector $\mathbf{Q}$. Choosing $\mathbf{Q}$ parallel or perpendicular to the direction of an external magnetic field $\mathbf{H}$ the direction dependent intermediate scattering function is measured by means of $\mathrm{x}$-ray photon correlation spectroscopy. From the intermediate scattering function in both directions the principal diffusion constants $D_{\|}$and $D_{\perp}$ are determined. The ratio $D_{\|} / D_{\perp}$ increases with increasing aspect ratio of the particles and can be described via a rescaled theoretical approach for prolate ellipsoids of revolution.
\end{abstract}

In recent decades, suspensions consisting of colloidal particles attracted a lot of interest as model systems for condensed matter at enlarged scales of space and time compared to molecular systems [1]. Due to the accessibility of highly defined spherical particles of both charged particles and hard sphere systems a variety of structural and dynamic phenomena resulting from the selforganization of such particles have been investigated $[2,3]$. However, because of the limited access to well defined anisotropic particles with a tunable aspect ratio only a few systems consisting of such particles have been investigated. Anisotropic particles, however, should give rise to a phase behavior with enhanced complexity compared to spherical particles due to the orientational degree of freedom [4-10]. Because of particle interactions or interactions with an external field such systems are expected to form analogs to liquid crystalline, i.e., nematic or smectic phases. While most of the existing studies address structural properties, less is known about the dynamic behavior of anisotropic particles. Several studies combining polarized and depolarized light scattering are published that give access both to the averaged translational and to rotational diffusion coefficients [11-15]. For ellipsoids and spherocylinders, with the averaged translational and rotational diffusion coefficients, the principal components $D_{\|}$and $D_{\perp}$ of the translational diffusion tensor can be calculated. The aim of this contribution is to investigate the translational diffusion coefficients $D_{\|}$and $D_{\perp}$ from particles aligned in an external field by directly probing the diffusive motion parallel and perpendicular to the particle axis.

Ozaki et al. [16] describe a method to produce spindleshaped particles consisting of hematite $\left(\alpha-\mathrm{Fe}_{2} \mathrm{O}_{3}\right)$ with a tunable aspect ratio. The resulting particles are single crystals with the long axis parallel to the trigonal axis of the hematite structure. Because of their canted antiferromagnetism the magnetic susceptibility of those particles is larger in the direction perpendicular than parallel to the trigonal axis resulting in a negative anisotropy of the magnetic susceptibility tensor with $\Delta \chi=\chi_{\|}-\chi_{\perp}<0$. In moderate magnetic fields with flux densities in the order of some $10^{-2} \mathrm{~T}$ these particles align with the long axis perpendicular to the direction of the external field [17].

X-ray photon correlation spectroscopy (XPCS) using the partial coherence of radiation emitted by third generation synchrotron sources is a powerful method to investigate slow processes in soft matter such as colloidal suspensions [18-20], gels [21,22], or polymers [23-25]. XPCS is not affected by multiple scattering or opacity for visible light. For magnetic particles with a strong absorption for optical wavelengths, XPCS is the only method to access the slow diffusive motion of such particles [26-28]. Because X-ray wavelengths are several orders of magnitude smaller than that of visible light, there is practically no limitation for the maximum accessible scattering vector $\left(Q_{\max }=4 \pi / \lambda\right)$, and, in addition, relevant length scales for colloidal systems can be probed by small scattering angles in the range less than 1 degree. In such a small-angle scattering 
geometry, the scattering vector is practically perpendicular to the primary beam. With a magnetic field perpendicular to the primary beam, a field in and perpendicular to the direction of the scattering vector can easily be applied.

In the absence of particle interactions and a drift induced by external fields, for anisotropic particles the diffusive motion can be described by the Fokker-Planck equation as

$$
\nabla_{r}^{T} \cdot \underline{\underline{\mathbf{D}}} \cdot \nabla_{r} P(\mathbf{r}, t)=\frac{\partial}{\partial t} P(\mathbf{r}, t)
$$

with $\underline{\underline{\mathbf{D}}}$ denoting the diffusion tensor of a particle and $P(\mathbf{r}, t)$ the normalized probability density to find a particle's center of mass at the time $t$ at the position $\mathbf{r}$. In highly dilute suspensions without particle interactions $\underline{\underline{\mathbf{D}}}$ equals the Stokes-Einstein self-diffusion tensor. If the particle is a body of revolution, this diffusion tensor reads as

$$
\underline{\underline{\mathbf{D}}}=\left[\begin{array}{lll}
D_{\perp} & & \\
& D_{\perp} & \\
& & D_{\|}
\end{array}\right]
$$

for a particle with the axis of revolution parallel to the $z$ direction. In Fourier space Eq. (1) can be written as

$$
-Q^{2} \hat{\mathbf{Q}}^{T} \cdot \underline{\underline{\mathbf{D}}} \cdot \hat{\mathbf{Q}} P(\mathbf{Q}, t)=\frac{\partial}{\partial t} P(\mathbf{Q}, t)
$$

with the unit vector $\hat{\mathbf{Q}}$ denoting the orientation of the scattering vector to the particles' axis of revolution.

For an ensemble of particles with an orientational distribution function (ODF) $f(\hat{\mathbf{u}})$ the Fokker-Planck equation reads

$$
-\frac{1}{Q^{2}} \frac{\partial}{\partial t} P(\mathbf{Q}, t)=\mathcal{L}(\hat{\mathbf{Q}}) P(\mathbf{Q}, t)
$$

with $\mathcal{L}(\hat{\mathbf{Q}})$ denoting the average diffusion coefficient over all orientations $\hat{\mathbf{u}}$ with respect to the $z$ direction of the laboratory coordinate system weighted by the $\operatorname{ODF} f(\hat{\mathbf{u}})$. This leads to the intermediate scattering function

$$
P(\mathbf{Q}, t)=P(\mathbf{Q}, 0) \exp \left[-Q^{2} \mathcal{L}(\hat{\mathbf{Q}}) t\right] .
$$

The average diffusion coefficient $\mathcal{L}(\hat{\mathbf{Q}})$ is given by

$$
\mathcal{L}(\hat{\mathbf{Q}})=\left\langle f(\hat{\mathbf{u}})[\underline{\underline{\mathbf{R}}}(-\hat{\mathbf{u}}) \cdot \hat{\mathbf{Q}}]^{T} \cdot \underline{\underline{\mathbf{D}}} \cdot[\underline{\underline{\mathbf{R}}}(-\hat{\mathbf{u}}) \cdot \hat{\mathbf{Q}}]\right\rangle_{\hat{\mathbf{u}}} \cdot
$$

Here, the quantity $\underline{\underline{\mathbf{R}}}(-\hat{\mathbf{u}})$ is a matrix that rotates the scattering unit vector $\hat{\mathbf{Q}}$ by $-\hat{\mathbf{u}}$. This corresponds to the rotation of the particle by $\hat{\mathbf{u}}$ within the coordinate system defined by the unit scattering vector $\hat{\mathbf{Q}}$. The rotation matrix $\underline{\underline{\mathbf{R}}}(\hat{\mathbf{u}})=\underline{\underline{\mathbf{R}}}(\vartheta, \varphi)=\underline{\underline{\mathbf{\Phi}}}(\varphi) \cdot \underline{\underline{\boldsymbol{\Theta}}}(\vartheta)$ combined of a rotation by $\vartheta$ around the $x$ direction and a second rotation by $\varphi$ around the $z$ direction reads as

$$
\underline{\underline{R}}(\vartheta, \varphi)=\left(\begin{array}{ccc}
\cos \varphi \cos \vartheta & -\sin \varphi & \cos \varphi \sin \vartheta \\
\sin \varphi \cos \vartheta & \cos \vartheta & \sin \varphi \sin \vartheta \\
-\sin \vartheta & 0 & \cos \vartheta
\end{array}\right) .
$$

In the absence of an external magnetic field, in dilute suspensions, the orientation of the particles is random. In this case, the ODF is given by

$$
f(\vartheta, \varphi)=\frac{1}{4 \pi}
$$

leading to the average diffusion coefficient

$$
\mathcal{L}(\hat{\mathbf{Q}})=\frac{1}{3} D_{\|}+\frac{2}{3} D_{\perp}
$$

for arbitrary orientations of the unit scattering vector $\hat{\mathbf{Q}}$ with respect to the laboratory coordinate system. Already for moderate flux densities in the range of several tens of militesla the particles are completely aligned perpendicular to the field direction corresponding to a deltashaped ODF

$$
f(\vartheta, \varphi)=\frac{1}{2 \pi} \delta\left(\vartheta-\frac{\pi}{2}\right)
$$

with respect to the direction of the magnetic field ( $z$ direction in the laboratory coordinate system) [17]. Now the quantity $\mathcal{L}(\hat{\mathbf{Q}})$ differs for unit scattering vectors parallel $\left(\hat{\mathbf{Q}}_{\| \hat{\mathbf{H}}}\right)$ and perpendicular $\left(\hat{\mathbf{Q}}_{\perp \hat{\mathbf{H}}}\right)$ to the field direction given by

$$
\hat{\mathbf{Q}}_{\| \hat{\mathbf{H}}}=\left(\begin{array}{l}
0 \\
0 \\
1
\end{array}\right) \quad \hat{\mathbf{Q}}_{\perp \hat{\mathbf{H}}}=\left(\begin{array}{c}
\cos \varphi \\
\sin \varphi \\
0
\end{array}\right)
$$

with $\vartheta=0$ and $\vartheta=\pi / 2$, respectively. With the delta shaped ODF Eq. (10) we obtain

$$
\begin{gathered}
\mathcal{L}\left(\hat{\mathbf{Q}}_{\| \hat{\mathbf{H}}}\right)=D_{\perp} \\
\mathcal{L}\left(\hat{\mathbf{Q}}_{\perp \hat{\mathbf{H}}}\right)=\frac{1}{2} D_{\|}+\frac{1}{2} D_{\perp} .
\end{gathered}
$$

As a consequence, for scattering vectors parallel to the field direction, the diffusion coefficient is $D_{\perp}$; for scattering vectors perpendicular to the field direction the average diffusion coefficient $\left(D_{\|}+D_{\perp}\right) / 2$ can be observed.

In the absence of particle interactions, i.e., in highly dilute suspensions with large interparticle distances, the field autocorrelation function $g_{1}(\mathbf{Q}, t)$ can be, in the limit of Gaussian diffusion, expressed as

$$
g_{1}(\mathbf{Q}, t)=\left\langle\exp \left\{-\imath \mathbf{Q} \cdot\left[\mathbf{r}_{i}(t)-\mathbf{r}_{j}(0)\right]\right\}\right\rangle,
$$

which is, for ergodic systems, connected to the intensity autocorrelation function $g_{2}(\mathbf{Q}, t)$ via the Siegert relation [29]

$$
g_{1}(\mathbf{Q}, t)=\left(\frac{g_{2}(\mathbf{Q}, t)-1}{\alpha(\mathbf{Q})}\right)^{1 / 2},
$$

where $\alpha(\mathbf{Q})$ denotes the contrast that is influenced by the coherence properties of the incident radiation and the geometry of the scattering experiment. 
Colloidal hematite particles are prepared according to the method described by Ozaki et al. [16]. The resulting spindle-shaped particles are characterized via transmission electron microscopy (Fig. 1) and small-angle x-ray scattering under a variation of an external magnetic field. These experiments, carried out at the high brilliance beam line ID02 of the European Synchrotron Radiation Facility (ESRF; Grenoble, France), show an orientation of the particles perpendicular to an external magnetic field due to their negative anisotropy of the magnetic susceptibility already at moderate flux densities in the range of several $10 \mathrm{mT}$ [17]. In highly dilute samples with a volume fraction $\phi \approx 10^{-3}$ containing $10^{-3} \mathrm{~mol} \mathrm{~L}^{-1} \mathrm{KCl}$ to screen surface charges, particle-particle interactions are not observed; i.e., the mesoscale structure of the colloidal system is the one of an ideal gas with $S(Q) \equiv 1$. Under these conditions free Gaussian diffusion takes place.

The XPCS experiments are carried out at the beam line ID10A (ESRF) with a beam focused to the sample position by beryllium lenses at a fixed incident energy of $8 \mathrm{keV}$. Higher harmonics of the undulator are cut off using a mirror. The beam size is $10 \times 10 \mu \mathrm{m}^{2}$. As a detector an avalanche photo diode with an aperture of $50 \times 50 \mu \mathrm{m}^{2}$ at a distance of $2.20 \mathrm{~m}$ to the sample is used resulting in typical contrast values of about 0.10 to 0.15 . In this configuration, a speckle size of approximately $30 \mu \mathrm{m}$ is achieved. Both the scattered signal and the monitor signal are correlated employing a FLEX correlator. The samples, sealed in $0.7 \mathrm{~mm}$ quartz capillaries, are positioned in a cell equipped with two rare earth permanent magnets with a pole size of $60 \times 37 \mathrm{~mm}^{2}$ and a pole distance of $65 \mathrm{~mm}$.

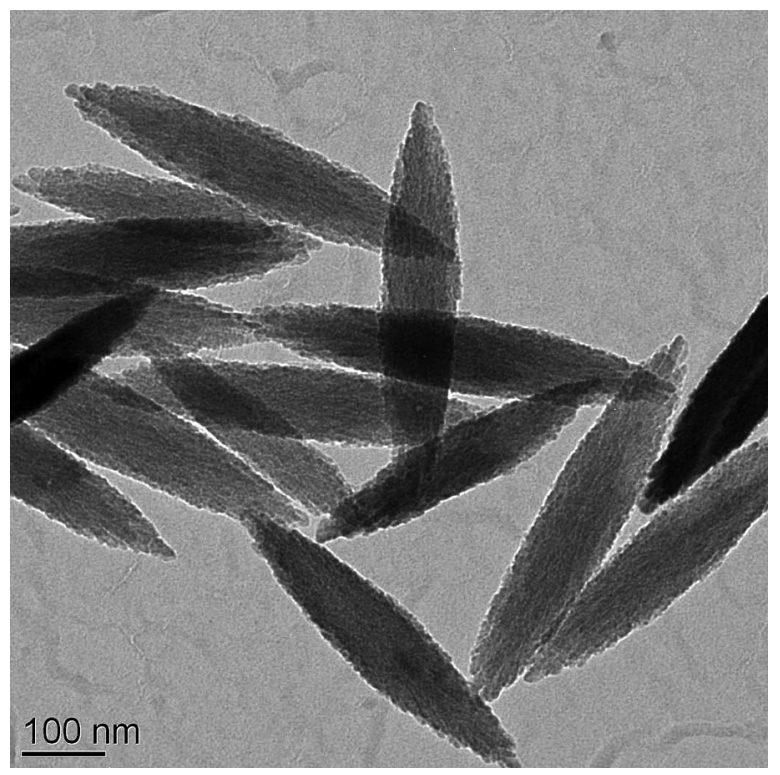

FIG. 1. Transmission electron micrograph of spindle-shaped hematite particles with an aspect ratio of $\nu=5.2 \pm 0.9$. The mean equatorial diameter is $\langle\sigma\rangle=(80 \pm 1) \mathrm{nm}$ with a polydispersity of $p_{\sigma}=0.12 \pm 0.04$.
With this setup a highly homogenous magnetic field with a flux density of $B_{0}=0.12 \mathrm{~T}$ is achieved. In the presence of this external magnetic field correlation functions are measured in a range of $9.3 \times 10^{-3} \mathrm{~nm}^{-1}<Q<2.8 \times$ $10^{-2} \mathrm{~nm}^{-1}$ in the directions $\mathbf{Q} \| \mathbf{H}$ and $\mathbf{Q} \perp \mathbf{H}$. To eliminate correlations resulting from the time structure of the incident beam, the intensity correlation function of the scattered signal is divided by that of the monitor.

A typical set of intensity correlation functions versus the modulus of the scattering vector $Q$ is displayed in Fig. 2 together with fits of the relaxation rates assuming a single exponential decay for the aspect ratio of $\nu=5.2 \pm 0.9$ without external field.

As the particles align with the long axis perpendicular to an external field, the diffusive motion in field direction is determined by $D_{\perp}$. Because of a free rotation around the short particle axis, for a motion perpendicular to the field an average $\left(D_{\|}+D_{\perp}\right) / 2$ is observed: the probability of finding a particle with its long axis parallel to the scattering vectors equals that of finding a particle with the long axis perpendicular to the scattering vector. With $D_{\perp}$ and $\left(D_{\|}+D_{\perp}\right) / 2$ both components of the diffusion tensor $\underline{\underline{\mathbf{D}}}$ are known.

In Figs. 3 and 4 the first cumulants of the field autocorrelation functions are displayed versus $Q^{2}$. The slope of the line of best fit equals the averaged Einstein diffusion coefficient for a motion parallel to the direction of $\mathbf{Q}$. The averaged diffusion coefficients without field are

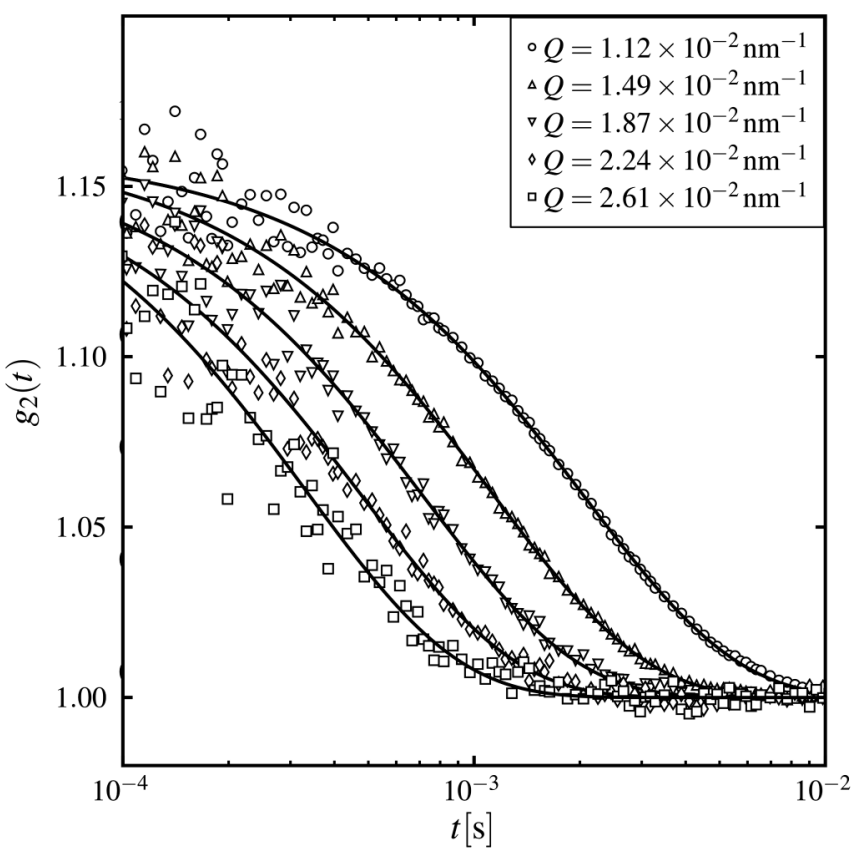

FIG. 2. Intensity autocorrelation functions obtained for particles with $\nu=5.2 \pm 0.9$ without external field normalized to the autocorrelation function of the monitor channel for increasing scattering vectors. The solid lines are fits of a single exponential decay. 


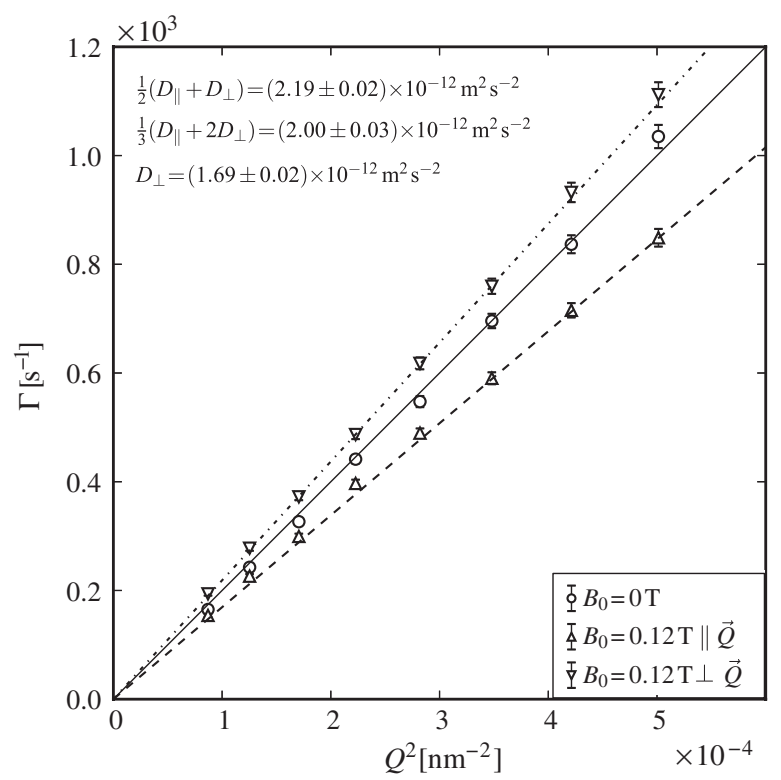

FIG. 3. First cumulants of the field autocorrelation function for hematite spindles with an aspect ratio $\nu=5.2 \pm 0.9$ versus $Q^{2}$ without external field (circles) and in the presence (triangles) of an external field. Because the particles are aligned with the long axis perpendicular to the field, in this direction the diffusion coefficient given by the slope of the curve $(\triangle)$ is smaller than in the orthogonal direction $(\nabla)$.

similar to the ones for ellipsoidal particles of comparable size found in the literature $[14,15]$.

With increasing aspect ratio the ratio $D_{\|} / D_{\perp}$ increases, too. The friction coefficients for ellipsoids are derived by Perrin [30] and further developed by Shimizu [31]. For prolate ellipsoids of revolution with the aspect ratio $\nu$ this quantity can be expressed as

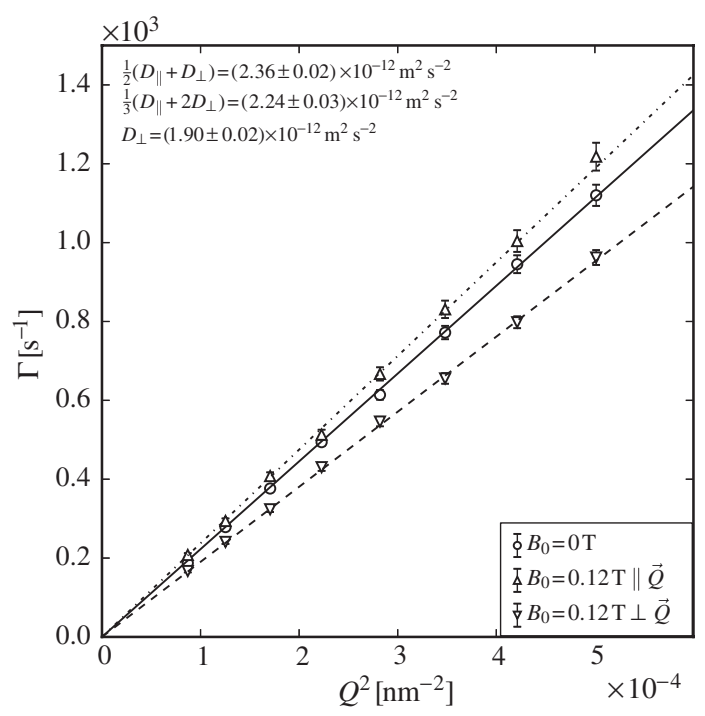

FIG. 4. First cumulants of the field autocorrelation function for hematite spindles with an aspect ratio $\nu=4.0 \pm 0.5$ versus $Q^{2}$ without external field (circles) and in the presence (triangles) of an external field.

$$
R(\nu)=\frac{D_{\|}}{D_{\perp}}=2 \frac{\left(2 \nu^{2}-1\right) S(\nu)-2 \nu}{\left(2 \nu^{2}-3\right) S(\nu)+2 \nu}
$$

with

$$
S(\nu)=\frac{2}{\left(\nu^{2}-1\right)^{1 / 2}} \ln \left[\nu+\left(\nu^{2}-1\right)^{1 / 2}\right]
$$

In Fig. 5 the experimental ratios $D_{\|} / D_{\perp}$ for several aspect ratios are compared to the theoretical prediction for ellipsoids by Perrin. For spindle-shaped particles as studied here, an increased diffusion coefficient $D_{\|}$can be expected due to a smaller surface than an ellipsoid of revolution with the same equatorial diameter and aspect ratio. The ratio $D_{\|} / D_{\perp}$ increases faster than predicted for prolate ellipsoids of revolution. The dashed line is a rescaled Perrin approach according to $1+(1.78 \pm 0.09)[R(\nu)-1]$ that very well describes the data within the experimental accuracy.

If anisotropic particles such as prolate or oblate ellipsoids or spherocylinders carry a magnetic moment or exhibit an anisotropic magnetic susceptibility, an external magnetic field is well suited to manipulate the orientation of such particles. In case of charged particles, due to the electric double layer, an electrostatic field can be used instead. In such cases, by appropriate choice of the scattering vectors $\mathbf{Q}$ with respect to the direction of an external field, direction dependent translational diffusion coefficients can directly be measured. The small-angle scattering geometry used to probe characteristic colloidal length scales easily allows us to realize sample environments to apply external magnetic or electric fields parallel and perpendicular to the scattering vector $\mathbf{Q}$. For light scattering experiments such a sample environment is difficult to realize due to the large scattering angles required to probe

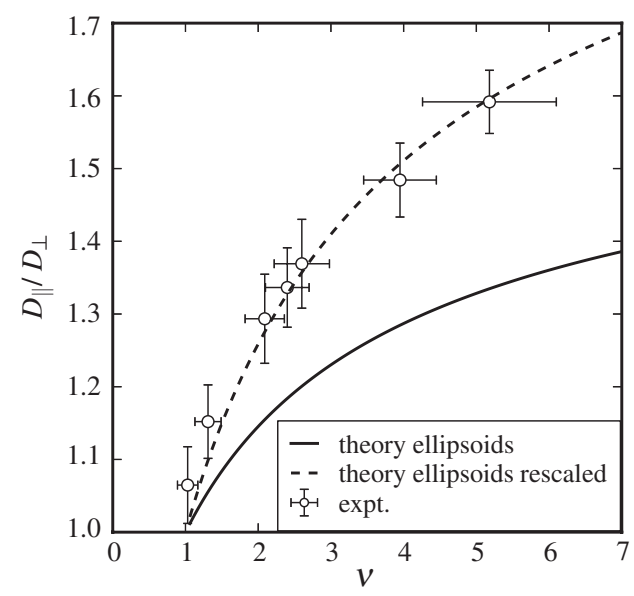

FIG. 5. Ratio of diffusion coefficients $D_{\|} / D_{\perp}$ in dependence on the aspect ratio determined by transmission electron microscopy. The theoretical result for prolate ellipsoids of revolution is displayed by the solid line. The dashed line represents a rescaled version of this theoretical approach. This rescaled theory very well describes the diffusive behavior of spindles. 
characteristic colloidal length scales. An additional advantage of XPCS is the applicability for optically opaque samples, where the opacity either results from multiple scattering or strong absorption for visible light as is the case for magnetic materials.

The diffusion tensor for anisotropic particles and the related friction coefficients are important to understand the rheological properties of colloidal suspensions consisting of anisotropic particles. By aligning anisotropic particles in an external field, the macroscopic, rheological properties of such suspensions will also change.

The difference $D_{\|}-D_{\perp}$ induces a coupling between translational and rotational diffusion [32] that needs to be considered for depolarized light scattering experiments. Employing XPCS, the translational diffusion coefficients $D_{\|}$and $D_{\perp}$ of aligned particles can directly be probed without the influence of the coupling of translational and rotational motion.

*joachim.wagner@uni-rostock.de

[1] P. N. Pusey, Colloidal Suspensions in Liquids, Freezing and Glass Transition (Elsevier, New York, 1991).

[2] P. N. Pusey and W. van Megen, Nature (London) 320, 340 (1986).

[3] P. N. Pusey and W. van Megen, Phys. Rev. Lett. 59, 2083 (1987).

[4] G. J. Vroege and H. N. W. Lekkerkerker, Rep. Prog. Phys. 55, 1241 (1992).

[5] B. J. Lemaire, P. Davidson, J. Ferré, J. P. Jamet, P. Panine, I. Dozov, and J. P. Jolivet, Phys. Rev. Lett. 88, 125507 (2002).

[6] B. J. Lemaire, P. Davidson, P. Panine, and J. P. Jolivet, Phys. Rev. Lett. 93, 267801 (2004).

[7] B. J. Lemaire, P. Davidson, D. Petermann, P. Panine, I. Dozov, D. Stoenescu, and J. P. Jolivet, Eur. Phys. J. E 13, 309 (2004).

[8] B. J. Lemaire, P. Davidson, J. Ferré, J.P. Jamet, D. Petermann, P. Panine, I. Dozov, and J.P. Jolivet, Eur. Phys. J. E 13, 291 (2004).

[9] M. P. B. van Bruggen, F. M. van der Kooij, and H. N. W. Lekkerkerker, J. Phys. Condens. Matter 8, 9451 (1996).
[10] J. A. N. Zasadzinski and R. B. Meyer, Phys. Rev. Lett. 56, 636 (1986).

[11] S. R. Aragon and R. Pecora, J. Chem. Phys. 66, 2506 (1977).

[12] D. Lehner, H. Lindner, and O. Glatter, Langmuir 16, 1689 (2000).

[13] J. Rodríguez-Fernández, J. Pézez-Juste, L. M. Liz-Marzán, and P. R. Lang, J. Phys. Chem. C 111, 5020 (2007).

[14] M. Hoffmann, C.S. Wagner, L. Harnau, and A. Wittemann, ACS Nano 3, 3326 (2009).

[15] I. Martchenko, H. Dietsch, C. Moitzi, and P. Schurtenberger, J. Phys. Chem. B 115, 14838 (2011).

[16] M. Ozaki, S. Kratohvil, and E. Matijevic, J. Colloid Interface Sci. 102, 146 (1984).

[17] C. Märkert, B. Fischer, and J. Wagner, J. Appl. Crystallogr. 44, 441 (2011).

[18] M. Sikorski, A. R. Sandy, and S. Narayanan, Phys. Rev. Lett. 106, 188301 (2011).

[19] M. Spannuth, S. G. J. Mochrie, S. S. L. Peppin, and J. S. Wettlaufer, J. Chem. Phys. 135, 224706 (2011).

[20] W. R. Burghardt, M. Sikorski, A. R. Sandy, and S. Narayanan, Phys. Rev. E 85, 021402 (2012).

[21] D. Orsi, L. Cristofolini, M. P. Fontana, E. Pontecorvo, C. Caronna, A. Fluerasu, F. Zontone, and A. Madsen, Philos. Mag. 91, 1836 (2011).

[22] D. Orsi, L. Cristofolini, G. Baldi, and A. Madsen, Phys. Rev. Lett. 108, 105701 (2012).

[23] P. Falus, M. A. Borthwick, and S. G. J. Mochrie, Phys. Rev. Lett. 94, 016105 (2005).

[24] A. J. Patel, S. Narayanan, A. Sandy, S. G. J. Mochrie, B. A. Garetz, H. Watanabe, and N. P. Balsara, Phys. Rev. Lett. 96, 257801 (2006).

[25] H. Guo, G. Bourret, R. B. Lennox, M. Sutton, J. L. Harden, and R. L. Leheny, Phys. Rev. Lett. 109, 055901 (2012).

[26] G. Grübel and F. Zontone, J. Alloys Compd. 362, 3 (2004).

[27] A. Robert, J. Wagner, T. Autenrieth, W. Härtl, and G. Grübel, J. Chem. Phys. 122, 084701 (2005).

[28] A. Robert, J. Wagner, T. Autenrieth, W. Härtl, and G. Grübel, J. Magn. Magn. Mater. 289, 47 (2005).

[29] B. J. Berne and R. Pecora, Dynamic Light Scattering: With Applications to Chemistry, Biology and Physics (Dover, Mineola, 2000), ISBN 0486411559.

[30] F. Perrin, J. Phys. Radium 5, 497 (1934).

[31] H. Shimizu, J. Chem. Phys. 37, 765 (1962).

[32] J. K. G. Dhont, An Introduction to Dynamics of Colloids (Elsevier, New York, 1996), ISBN 0444820094. 